\title{
M
}

GMR

\section{Predictive potential role of glutathione S-transferases polymorphisms in response to chemotherapy and breast cancer prognosis}

\author{
P. Yuan', L. Yuan², B.L. Xü, C.Z. Wang ${ }^{1}$, H.Z. Yang ${ }^{1}$ and Y. Li ${ }^{4}$ \\ ${ }^{1}$ Breast Cancer Center, \\ The Affiliated Cancer Hospital of Zhengzhou University, Zhengzhou, China \\ 2Department of Surgery, \\ The Affiliated Cancer Hospital of Zhengzhou University, Zhengzhou, China \\ ${ }^{3}$ Central Laboratory, \\ The Affiliated Cancer Hospital of Zhengzhou University, Zhengzhou, China \\ ${ }^{4}$ Department of Thoracic Surgery, \\ The Affiliated Cancer Hospital of Zhengzhou University, Zhengzhou, China \\ Corresponding author: $\mathrm{Y}$. $\mathrm{Li}$ \\ E-mail: liyin_yin5@163.com
}

Genet. Mol. Res. 14 (4): 16675-16681 (2015)

Received August 27, 2015

Accepted October 2, 2015

Published December 11, 2015

DOI http://dx.doi.org/10.4238/2015.December.11.15

ABSTRACT. The aim of this study was to evaluate the role of GSTM1 null/ present, GSTT1 null/present, and GSTP1 polymorphisms in the clinical response to chemotherapy and treatment outcome of breast cancer. The GSTM1, GSTT1, and GSTP1 Ile105Val polymorphism genotypes were analyzed using polymerase chain reaction coupled with restriction fragment length polymorphism. Conditional logistic regression analysis revealed that breast cancer patients carrying the GG genotype of GSTP1 lle105Val showed a significantly better response to chemotherapy compared to those expressing the AA genotype [odds ratio $=2.66,95 \%$ confidence interval $(\mathrm{Cl})=1.24-5.91, \mathrm{P}=0.007]$. The Cox proportional hazards model indicated 
that the GG genotype of GSTP1 Ile105Val in breast cancer patients was correlated with a lower risk of death from all causes than those with AA genotype. The adjusted hazard ratio $(95 \% \mathrm{Cl})$ for the $\mathrm{GG}$ genotype of GSTP1 Ile105Val was $0.44(0.18-0.99 ; P=0.03)$. In conclusion, the results of our study indicated that the GG genotype of GSTP1 Ile105Val was significantly associated with better response to chemotherapy and longer overall survival, compared to the wide-type genotype.

Key words: Glutathione S-transferases; Polymorphism; Chemotherapy; Breast cancer

\section{INTRODUCTION}

Breast cancer is, by far, the most frequent type of cancer affecting women, and the leading cause of malignancy-related deaths in many countries (Jemal et al., 2009). An estimated 1.67 million new cases of cancer have been diagnosed in 2012 , accounting for $25 \%$ of all cancers (IARC, 2012). Chemotherapy is an adjuvant systemic therapy administered after primary surgery, or a neo-adjuvant chemotherapy applied before surgery in patients with locally advanced breast cancers (van der Hage et al., 2001). Although many clinicopathological characteristics have been unable to precisely predict the efficacy of chemotherapy, increasing evidence has suggested that drug-metabolizing enzymes play an important role in determining inter-individual variations in therapeutic response (Arun et al., 2010).

GSTs detoxify chemotherapeutic drugs or their metabolites by catalyzing the reduction of these compounds with glutathione. GSTM1, GSTT1 and GSTP1 are three common enzymes belonging to the GST superfamily. Allelic deletions in the GSTM1 and GSTT1 genotypes are correlated with reduced enzyme production (Strange et al., 2001). Polymorphisms in GSTP1 are associated with lower substrate-specific catalytic activity. Previous studies have suggested that genetic polymorphisms in the GST genes could influence the effectiveness of detoxification of the cytotoxins generated by the chemotherapeutic agents used in breast cancer treatment (Bai et al., 2012; Tulsyan et al., 2013; Vivenza et al., 2013; Oliveira et al., 2014); however, these studies have yielded inconsistent results. Therefore, the aim of this study was to evaluate the role of GSTM1 null/present, GSTT1 null/present, and GSTP1 polymorphisms in the clinical response to chemotherapy and the outcome of breast cancer treatment.

\section{MATERIAL AND METHODS}

\section{Patients}

Two hundred and ninety-two subjects were recruited between April 2009 and April 2012 from the Affiliated Cancer Hospital of Zhengzhou University for this study. This cohort of breast cancer patients had been newly diagnosed, were histopathologically confirmed, and were untreated. Among these, 273 breast cancer patients agreed to participate in this study (participation rate, $93.49 \%$ ), and signed informed consent forms to this effect.

The clinical characteristics of the enrolled patients, including tumor size, clinical stages, 
lymph mode metastasis, and estrogen receptor (ER) and progesterone receptor (PR) status, were collected from the patient medical records. A standardized questionnaire was used to collect the demographic data, including mean age and menopausal status.

All 273 patients were treated with chemotherapy; these breast cancer patients received chemotherapy treatment evaluation based on the RECIST criteria (Duffaud and Therasse, 2000). Response to chemotherapy was stratified into the response (complete response or partial response) and non-response (stable disease or progressive disease) types. All patients were followed up until April 2014. the overall survival (OS) of breast cancer patients was defined as the time from the beginning of study (April 2009) to death. Breast cancer patients who were alive at the time of analysis were excluded from the study on the day of the final follow-up.

\section{DNA extraction and genotyping}

DNA was extracted from peripheral blood samples (collected from patients) using TIANamp Blood DNA kit (Tiangen Biotech Co., Ltd., Beijing, China). The GSTM1, GSTT1, and GSTP1 lle105Val genotypes were analyzed by polymerase chain reaction (PCR) coupled with restriction fragment length polymorphism (RFLP). The PCR fragments were subsequently digested with their specific restriction enzymes. The digestion products were separated by electrophoresis on an ethidium bromide stained agarose gel, and visualized under UV light.

\section{Statistical methods}

Statistical analyses were performed on a STATA v.9.0 software platform (StataCorp LP, College Station, TX, USA); statistical significance was determined by two-sided tests. Survival curves were analyzed by the Kaplan-Meier method to evaluate the impact of the GSTM1, GSTT1, and GSTP1 Ile105Val polymorphisms on OS. The association between the GSTM1, GSTT1, and GSTP1 Ile105Val polymorphisms and response to chemotherapy was analyzed by a conditional logistic regression method; this association was presented as the odds ratio (OR) and 95\% confidence interval (Cl). The association between the GSTM1, GSTT1, and GSTP1 Ile105Val polymorphisms and OS was analyzed by the Cox proportional hazards model; this association was expressed as the hazard ratio $(\mathrm{HR})$ with a $95 \% \mathrm{Cl}$. Two-sided $\mathrm{P}$ values $<0.05$ were considered to be statistically significant.

\section{RESULTS}

The clinical and demographic characteristics of 273 breast cancer patients are summarized in Table 1. The mean age of the inducted breast cancer patients was $55.7 \pm 11.4$ years. One hundred and forty six of the included patients $(53.48 \%)$ were premenopausal, and $127(46.52 \%)$ were postmenopausal. The tumor size of 96 patients $(35.16 \%)$ was $<2.0 \mathrm{~cm}$ and that of $177(64.84 \%)$ patients was $>2.0 \mathrm{~cm}$. One hundred and eighty two patients $(66.67 \%)$ were at clinical stages I-II, and 91 (33.33\%) were at stages III-IV. Furthermore, 153 patients (56.04\%) exhibited positive lymph node metastasis, and 120 (43.96\%) showed negative lymph mode metastasis. One hundred and sixty five patients (60.44\%) showed positive ER status, while 143 $(52.38 \%)$ showed positive PR status. 


\begin{tabular}{|c|c|c|}
\hline Characteristics & Number & $\%$ \\
\hline Mean age, years & $55.7 \pm 11.4$ & \\
\hline \multicolumn{3}{|l|}{ Age, years } \\
\hline$\leq 50$ & 122 & 44.69 \\
\hline$>50$ & 151 & 55.31 \\
\hline \multicolumn{3}{|l|}{ Menopausal status } \\
\hline Premenopausal & 146 & 53.48 \\
\hline Postmenopausal & 127 & 46.52 \\
\hline \multicolumn{3}{|l|}{ Tumor size, cm } \\
\hline$\leq 2.0$ & 96 & 35.16 \\
\hline$>2.0$ & 177 & 64.84 \\
\hline \multicolumn{3}{|l|}{ Clinical stages } \\
\hline I-II & 182 & 66.67 \\
\hline III-IV & 91 & 33.33 \\
\hline \multicolumn{3}{|c|}{ Lymph node metastasis } \\
\hline Negative & 120 & 43.96 \\
\hline Positive & 153 & 56.04 \\
\hline \multicolumn{3}{|l|}{ ER status } \\
\hline Negative & 108 & 39.56 \\
\hline Positive & 165 & 60.44 \\
\hline \multicolumn{3}{|l|}{ PR status } \\
\hline Negative & 128 & 46.89 \\
\hline Positive & 143 & 52.38 \\
\hline
\end{tabular}

At the end of the follow-up period, 169 breast cancer patients (61.90\%) exhibited CR + PR to chemotherapy, while $104(38.10 \%)$ showed a SD + PD status (Table 2). Conditional logistic regression analysis revealed that patients carrying the GG genotype of the GSTP1 Ile105Val polymorphism showed a significantly better response to chemotherapy, compared to the AA genotype $(\mathrm{OR}=2.66,95 \% \mathrm{Cl}=1.24-5.91, \mathrm{P}=0.007)$. However, we observed no significant association between the GSTM1 and GSTT1 polymorphisms and response to chemotherapy in breast cancer patients $(P>0.05)$.

Table 2. Association between the GSTM1, GSTT1, and GSTP1 lle105Val polymorphisms and response to chemotherapy.

\begin{tabular}{|c|c|c|c|c|c|c|c|c|}
\hline \multirow[t]{2}{*}{ Genotype } & \multirow[t]{2}{*}{ Patients } & \multirow[t]{2}{*}{$\%$} & \multicolumn{4}{|c|}{ Response to chemotherapy } & \multirow[t]{2}{*}{ OR $(95 \% \mathrm{Cl})^{1}$} & \multirow[t]{2}{*}{$P$ value } \\
\hline & & & Responder (CR+PR) & $\%$ & Non-responder (SD+PD) & $\%$ & & \\
\hline \multicolumn{9}{|l|}{ GSTM1 } \\
\hline Present & 156 & 57.14 & 93 & 55.03 & 63 & 60.58 & 1.0 (Ref.) & - \\
\hline Null & 117 & 42.86 & 76 & 44.97 & 41 & 39.42 & $1.26(0.74-2.13)$ & 0.37 \\
\hline \multicolumn{9}{|l|}{ GSTT1 } \\
\hline Present & 121 & 44.32 & 73 & 43.20 & 48 & 46.15 & 1.0 (Ref.) & - \\
\hline Null & 152 & 55.68 & 96 & 56.80 & 56 & 53.85 & $1.12(0.67-1.90)$ & 0.63 \\
\hline \multicolumn{9}{|c|}{ GSTP1 lle105Val } \\
\hline AA & 105 & 38.46 & 55 & 32.54 & 50 & 48.08 & 1.0 (Ref.) & - \\
\hline$A G$ & 113 & 41.39 & 73 & 43.20 & 40 & 38.46 & $1.66(0.93-2.96)$ & 0.07 \\
\hline GG & 55 & 20.15 & 41 & 24.26 & 14 & 13.46 & $2.66(1.24-5.91)$ & 0.007 \\
\hline
\end{tabular}

${ }^{1}$ Adjusted for age, menopausal status, tumor size, clinical stage, lymph node metastasis, ER status and PR status. OR = odds ratio; $\mathrm{Cl}=$ confidence interval.

Sixty-seven patients died during the follow-up period by the end of April 2014; the five year survival rate of breast cancer patients was calculated to be $24.54 \%$. The GG genotype of the GSTP1 Ile 105Val polymorphism in breast cancer patients was correlated with a lower risk of death from all causes by the Cox proportional hazards model, compared to the AA genotype (Table 3 ). 
The adjusted HR $(95 \% \mathrm{Cl})$ for the GG genotype of the GSTP1 lle105Val polymorphism was 0.44 (0.18-0.99) $(P=0.03)$. However, the GSTM1 and GSTT1 gene polymorphisms did not influence the overall survival of breast cancer patients $(P>0.05)$.

\begin{tabular}{|c|c|c|c|c|c|c|c|c|}
\hline Genotype & Patients & $\%$ & Event & $\%$ & Alive & $\%$ & $\mathrm{HR}(95 \% \mathrm{Cl})^{1}$ & $P$ value \\
\hline \multicolumn{9}{|l|}{ GSTM1 } \\
\hline Present & 156 & 57.14 & 39 & 58.21 & 114 & 55.34 & 1.0 (Ref.) & - \\
\hline Null & 117 & 42.86 & 28 & 41.79 & 92 & 44.66 & $0.89(0.49-1.61)$ & 0.68 \\
\hline \multicolumn{9}{|l|}{ GSTT1 } \\
\hline Present & 121 & 44.32 & 30 & 44.78 & 89 & 43.20 & 1.0 (Ref.) & - \\
\hline Null & 152 & 55.68 & 37 & 55.22 & 117 & 56.80 & $0.94(0.52-1.70)$ & 0.82 \\
\hline \multicolumn{9}{|c|}{ GSTP1 Ile105Val } \\
\hline AA & 105 & 38.46 & 28 & 41.79 & 59 & 28.64 & 1.0 (Ref.) & - \\
\hline AG & 113 & 41.39 & 27 & 40.30 & 89 & 43.20 & $0.64(0.33-1.25)$ & 0.16 \\
\hline GG & 55 & 20.15 & 12 & 17.91 & 58 & 28.16 & $0.44(0.18-0.99)$ & 0.03 \\
\hline
\end{tabular}

${ }^{1}$ Adjusted for age, menopausal status, tumor size, clinical stage, lymph node metastasis, ER status and PR status. $\mathrm{HR}=$ hazard ratio; $\mathrm{Cl}=$ confidence interval.

\section{DISCUSSION}

In this study, we evaluated the association between the GSTM1, GSTT1, and GSTP1 Ile105Val polymorphisms and the response to chemotherapy in, and overall survival of, breast cancer patients in a Chinese population. The results of our study indicated that the GG genotype of GSTP1 lle105Val was associated with good response to chemotherapy, and was correlated with high overall survival of breast cancer.

The GST super-family of enzymes is a part of the phase Il group of enzymes; GST enzymes play an important role in the metabolism of many xenobiotics and drugs, including cytotoxic cancer chemotherapeutic agents (Tew, 1994). Many previous studies have reported that glutathione S-transferases may play an important role in determining the efficacy of chemotherapy on cancer (Ruwali et al., 2011; Suneetha et al., 2011; Bai et al., 2012; Goričar et al., 2015); however, the results of subsequent studies have shown the inconsistent nature of this relationship.

Recent studies have investigated the association of genetic polymorphisms in the GSTM1, GSTT1, and GSTP1 lle105Val region with breast cancer prognosis. People with variant GST genotypes display a reduced ability to detoxify drug metabolites, thereby ensuring a longer overall breast cancer survival. Several previous studies have reported that GSTs play a role in the prognosis of breast cancer patients subjected to chemotherapy (Syamala et al., 2008; Gor et al., 2010; Zhang et al., 2010; Bai et al., 2012; Romero et al., 2012; Zhou et al., 2015). Zhang et al. (2010), in a study conducted in a Chinese population, reported an association between polymorphisms in the GSTP1 gene and good response and light toxicity in breast cancer patients. Bai et al. (2012), who also conducted a study in a Chinese population, reported that null GSTM1 and GSTP1 Val/Val genotypes showed significantly better response rates to chemotherapy, compared to wide-type genotypes. On the other hand, Romero et al. (2012) discovered an association between the GSTP1 polymorphism and a lower risk of chemo-resistance, when treated with doxorubicin. However, some inconsistent results have also been reported. Gor et al. (2010) did not find a significant association between GST gene polymorphisms and disease-free survival and overall survival in breast cancer patients. Syamala et al. (2008), in a study conducted in an Indian population, reported that polymorphisms 
in GST genes did not affect the overall survival of sporadic breast cancer patients, while Zhou et al. (2015), in a study with 420 breast cancer patients, reported that the GSTP1 Ile105Val polymorphism was associated with an increased risk of death from breast cancer and poor tumor response to chemotherapy. These inconsistency results may be attributed to the differences in ethnicities and source of patients, as well as the disease stages and sample size.

There were several limitations to our study: the breast cancer patients were selected from one hospital only, which may cause selection bias. The sample size used for the analysis of association between GST polymorphisms and breast cancer survival was relatively small; therefore, some of the findings may be undervalued because of the limited number of studies available for analyses. Therefore, studies with larger sample sizes must be performed to confirm the results obtained in this study.

In conclusion, the results of our study indicated that the GG genotype of the GSTP1 Ile105Val polymorphism was significantly associated with better response to chemotherapy and longer overall survival, compared to the wide-type genotype. Further prospective studies with larger sample sizes are required to validate this association.

\section{Conflicts of interest}

The authors declare no conflict of interest.

\section{ACKNOWLEDGMENTS}

Research supported by the Basic and Advanced Technology Research Project of Henan Province, China (\#132300410041) and the Henan Medical Science and Technique Foundation (\#201401016). We acknowledge the help from nurses in the Affliated Cancer Hospital of Zhengzhou University, who helped us to collect the blood samples.

\section{REFERENCES}

Arun BK, Granville LA, Yin G, Middleton LP, et al. (2010). Glutathione-s-transferase-pi expression in early breast cancer: association with outcome and response to chemotherapy. Cancer Invest. 28: 554-559.

Bai YL, Zhou B, Jing XY, Zhang B, et al. (2012). Predictive role of GSTs on the prognosis of breast cancer patients with neoadjuvant chemotherapy. Asian Pac. J. Cancer Prev. 13: 5019-5022.

Duffaud $\mathrm{F}$ and Therasse P (2000). New guidelines to evaluate the response to treatment in solid tumors. Bull. Cancer 87: 881-886.

Fisher B, Bryant J, Wolmark N, Mamounas E, et al. (1998). Effect of preoperative chemotherapy on the outcome of women with operable breast cancer. J. Clin. Oncol. 16: 2672-2685.

Goričar K, Kovač V, Jazbec J, Zakotnik B, et al. (2015). Genetic variability of DNA repair mechanisms and glutathione-Stransferase genes influences treatment outcome in osteosarcoma. Cancer Epidemiol. 39: 182-188.

Gor PP, Su HI, Gray RJ, Gimotty PA, et al. (2010). Cyclophosphamide-metabolizing enzyme polymorphisms and survival outcomes after adjuvant chemotherapy for node-positive breast cancer: a retrospective cohort study. Breast Cancer Res. 12: R26.

International Agency for Research on Cancer (IARC). GLOBOCAN 2012: Estimated Cancer Incidence, Mortality and Prevalence Worldwide in 2012. Available at [http://globocan.iarc.fr/Pages/fact_sheets_cancer.aspx]. Accessed August 1, 2015.

Jemal A, Siegel R, Ward E, Hao Y, et al. (2009). Cancer statistics, 2009. CA. Cancer J. Clin. 59: 225-249.

Oliveira AL, Oliveira Rodrigues FF, Dos Santos RE, Rozenowicz RL, et al. (2014). GSTT1, GSTM1, and GSTP1 polymorphisms as a prognostic factor in women with breast cancer. Genet. Mol. Res. 13: 2521-2530.

Romero A, Martín M, Oliva B, de la Torre J, et al. (2012). Glutathione S-transferase P1 c.313A \&gt; G polymorphism could be useful in the prediction of doxorubicin response in breast cancer patients. Ann. Oncol. 23: 1750-1756. 
Ruwali M, Singh M, Pant MC and Parmar D (2011). Polymorphism in glutathione S-transferases: susceptibility and treatment outcome for head and neck cancer. Xenobiotica 41: 1122-1130.

Strange RC and Fryer AA (1999). The glutathione S-transferases: influence of polymorphism on cancer susceptibility. IARC Sci. Publ. 148: 231-249.

Strange RC, Spiteri MA, Ramachandran S and Fryer AA (2001). Glutathione-S-transferase family of enzymes. Mutat. Res. 482: 21-26.

Suneetha KJ, Nancy KN, Rajalekshmy KR, Rama R, et al. (2011). Role of glutathione-s-transferase and CYP1A1*2A polymorphisms in the therapy outcome of south Indian acute lymphoblastic leukemia patients. Indian J. Med. Paediatr. Oncol. 32: 25-29.

Tew KD (1994). Glutathione-associated enzymes in anticancer drug resistance. Cancer Res. 54: 4313-4320.

Tulsyan S, Chaturvedi P, Agarwal G, Lal P, et al. (2013). Pharmacogenetic influence of GST polymorphisms on anthracyclinebased chemotherapy responses and toxicity in breast cancer patients: a multi-analytical approach. Mol. Diagn. Ther. 17: 371-379.

van der Hage JA, van de Velde CJ, Julien JP, Tubiana-Hulin M, et al. (2001). Preoperative chemotherapy in primary operable breast cancer: results from the European Organization for Research and Treatment of Cancer trial 10902. J. Clin. Oncol. 19: 4224-4237.

Vivenza D, Feola M, Garrone O, Monteverde M, et al. (2013). Role of the renin-angiotensin-aldosterone system and the glutathione S-transferase $\mathrm{Mu}, \mathrm{Pi}$ and Theta gene polymorphisms in cardiotoxicity after anthracycline chemotherapy for breast carcinoma. Int. J. Biol. Markers 28: e336-e347.

Zhang BL, Sun T, Zhang BN, Zheng S, et al. (2011). Polymorphisms of GSTP1 is associated with differences of chemotherapy response and toxicity in breast cancer. Chin. Med. J. 124: 199-204.

Zhou L, Huang A, Zhang D, Yao J, et al. (2015). Genetic variability of glutathione S-transferases influences treatment outcome of breast cancer. Tumour Biol. 36: 5925-5929. 